\title{
Constant rate thermal analysis for thermal stability studies of polymers
}

Pedro E. Sánchez-Jiménez, Luis A. Pérez-Maqueda, Antonio Perejón, José M. Criado

Instituto de Ciencia de Materiales de Sevilla, C.S.I.C.-Universidad de Sevilla, C. Américo Vespucio no. 49, 41092 Sevilla, Spain

\begin{abstract}
This paper explores the relationship between the shapes of temperature-time curves obtained from experimental data recorded by means of constant rate thermal analysis (CRTA) and the kinetic model followed by the thermal degradation reaction. A detailed shape analysis of CRTA curves has been performed as a function of the most common kinetic models. The analysis has been validated with simulated data, and with experimental data recorded from the thermal degradation of polytetrafluoroethylene (PTFE), poly(1,4-butylene terephthalate) (PBT), polyethylene ( $\mathrm{PE}$ ) and poly(vinyl chloride) (PVC). The resulting temperature-time profiles indicate that the studied polymers decompose through phase boundary, random scission, diffusion and nucleation mechanisms respectively. The results here presented demonstrate that the strong dependence of the temperature-time profile on the reaction mechanism would allow the real kinetic model obeyed by a reaction to be discerned from a single CRTA curve.
\end{abstract}

\section{Keywords}

CRTA; Kinetic models; Thermal degradation; Kinetics; Random scission

\section{Introduction}

Thermal analysis techniques are routinely used in many scientific and industrial laboratories for studying the thermal decomposition of polymeric materials. Among them, thermogravimetry (TG) is one of the most common since the mass of a sample is easy to measure accurately and valuable information regarding the nature of the process can be extracted from a mass loss against time or temperature plot. While most TG studies are carried out under isothermal or linear heating rate conditions, constant rate thermal analysis (CRTA), formerly introduced by Rouquerol, constitutes a promising alternative [1] and [2] that has already proven its usefulness in sintering and preparation of materials [3], [4], [5], [6], [7], [8], [9], [10], [11], [12], [13] and [14]. While several papers dealing with the application of this method to the study of the kinetics of solid state reactions have been published [15], [16] and [17], this approach has rarely been extended into thermal degradation of polymers [18] and [19].

The main differences between CRTA and conventional methods are summarized in Fig. 1 and Fig. 2. Fig. 1 has been adapted from the one originally published by Reading [20] and [21] and shows the different trends the temperature, conversion and reaction rate follows with time under isothermal, linear heating and controlled rate methods. Alternatively, Fig. 2 illustrates the different setups needed for running conventional (isothermal and linear heating) and CRTA 
experiments. During a CRTA experiment, the temperature is controlled in such a way that the reaction rate is kept at a constant predefined value along the entire process [1], [2], [22], [23] and [24]. If the selected reaction rate is low enough, the mass and heat transfer phenomena that accompanies thermal decomposition processes can be minimized significantly, thereby yielding results which are much more representative of the forward reaction [1], [15], [25] and [26]. This advantage is especially useful in thermal degradation of polymers because many volatiles are usually evolved and may influence the evolution of the reaction [25]. Additionally, polymers have generally a low thermal conductivity and heat transfer phenomena can play a significant role in thermal degradation if experimental conditions are not carefully controlled. Furthermore, the enhanced control over the decomposition process provided by CRTA makes it a much more effective tool than conventional methods for the discrimination of overlapping processes. An additional feature of CRTA experiments is that the $\alpha-T$ plots are strongly dependant on the reaction mechanism. This property of CRTA methodology constitutes an important advantage over conventional TG. It has been well established that it is impossible to discriminate among kinetic models from a single TG curve when obtained under linear heating conditions since thermal degradation curves thus recorded are all sigmoidal in shape regardless of the reaction mechanism [27], [28], [29] and [30]. On the other hand, due to the close relationship between the temperature-time profile of CRTA curves and the reaction mechanism, it should be possible to unambiguously discern the kinetic model from a single CRTA curve.

Several kinetic models for the description of solid state reactions have been proposed during the last decades, with diffusion, phase boundary controlled and nucleation and growth of nuclei being the most usual ones [31] and [32]. These kinetic models account for the relationship between the reaction rate and the conversion $\alpha$ by assuming certain ideal geometrical conditions and reaction driving forces. Nevertheless, when studying polymer degradation reactions most authors tend to ignore these models, resorting instead to empirical first or n-order models that have no physical meaning and, even more important, give no guarantee they are actually representative of the studied process. However, recent works have demonstrated that those ideal models can often describe the thermal degradation of polymers much more precisely than "n-order" laws [33], [34], [35], [36], [37] and [38]. Furthermore, a new kinetic model for random scission, a mechanism to which the degradation of a wide range of polymers is attributed, has recently been developed [39].

The aim of this work is to carry out a detailed shape analysis of CRTA curves as a function of the different kinetic models and extend it to the recently proposed model for random scission reactions. Such work would be very illustrative of the power of the technique for discriminating the real kinetic model of the reaction from a single experimental curve. The shape analysis will be validated by a set of experimental CRTA curves obtained from the thermal decomposition of four commercial polymers: polytetrafluoroethylene (PTFE), poly(1,4butylene terephthalate) (PBT), polyethylene (PE) and poly(vinyl chloride) (PVC).

\section{Materials and methods}

Commercial Poly(butylene terephthalate) (Aldrich, product number 435147), polytetrafluoroethylene (Aldrich, product number 182478), polyethylene (Aldrich, product 
number 332119 , medium density $d=0.940$ ) and poly(vinyl chloride) (Aldrich 389323, average $\mathrm{Mn} \sim 47,000$; average $\mathrm{Mw} \sim 80,000$ ) were used in this work. Thermogravimetry measurements were carried out with a homemade TGA instrument that uses a $\mathrm{Cl}$ Electronics Ltd electrobalance connected to a gas flow system to work in inert atmosphere (70 cc min-1 N2). Small samples $(9 \mathrm{mg})$ were used in order to minimize heat and mass transfer phenomena. They were placed on a $1 \mathrm{~cm}$ diameter platinum pan inside a low thermal mass homemade furnace. The instrument allows working either under conventional linear heating conditions or under sample controlled conditions. A description of the experimental set-up is shown in Fig. $2 \mathrm{~b}$ and can also be found in more detail in references [22], [40] and [41].

\section{Shape analysis of CRTA curves}

The reaction rate, $d \alpha / d t$, of a solid state reaction can be described by the following equation:

$\frac{d \alpha}{d t}=A \exp (-E / R T) f(\alpha)$

where $\mathrm{A}$ is the Arrhenius pre-exponential factor, $\mathrm{R}$ is the gas constant, $\mathrm{E}$ the activation energy, $\alpha$ the reacted fraction, $T$ is the process temperature and $f(\alpha)$ accounts for the reaction rate dependence on $\alpha$. The kinetic model, $f(\alpha)$ is an algebraic expression which is usually associated with a physical model that describes the kinetics of the solid state reaction [32]. Table 1 shows the functions corresponding to the most common mechanisms in literature.

In the case of experiences carried out under constant rate conditions, the reaction rate, $d \alpha / d t$, is maintained at a constant value, C, during the entire experiment. Hence, Eq. (1) could be rewritten as:

$C=A e^{-E / R T} f(\alpha)$

\subsection{Diffusion, Avrami-Erofeev and "n-order" models}

As it was mentioned before, the shape of the CRTA curves is strongly dependant on the reaction mechanism. The shape analysis for the different kinetic models in Table 1 has been performed by means of determining the existence of minima, maxima or inflection points in the temperature versus $\alpha$ plots, and by locating at what $\alpha$ values those effects appear. From Eq. (2), after taking logarithms and rearranging terms we get:

$$
-\ln f(\alpha)=\ln \frac{A}{C}-\frac{E}{R T}
$$

The first and second derivatives of the temperature with respect to $\alpha$ result in the following expressions, Eqs. (4) and (5):

$$
\frac{d T}{d \alpha}=\frac{R T^{2}}{E_{a}} \frac{f(\alpha)}{f(\alpha)}
$$

$$
\frac{d^{2} T}{d \alpha^{2}}=-\frac{R T^{2}}{E_{a}}\left[\frac{2 R T}{E_{a}}\left(\frac{f^{\prime}(\alpha)}{f(\alpha)}\right)^{2}-\frac{f^{\prime}(\alpha) f(\alpha)-f(\alpha)^{2}}{f(\alpha)^{2}}\right]
$$


The first and second derivatives of the considered kinetic models, $f^{\prime}(\alpha)$ and $f^{\prime \prime}(\alpha)$, have been listed in Table 1 together with the corresponding $f(\alpha)$ functions. For a plot of $T$ against $\alpha$ to show a maximum or a minimum, a certain $\alpha \mathrm{m}$ at which $\mathrm{dT} / \mathrm{d} \alpha=0$ must exist. Therefore, at the maximum/minimum the following condition must be fulfilled:

$f\left(\alpha_{m}\right)=0$

The above equation has solution only for Avrami-Erofeev models when $n>1$. In such cases, a maximum/minimum appears at an $\alpha \mathrm{m}$ value which is given by the following equation:

$\alpha_{m}=1-\exp \left(\frac{1-n}{n}\right)$

According to Eq. (7), am depends only on the Avrami-Erofeev order. Therefore, from Eqs. (5) and (6) is easy to deduce that $\mathrm{d} 2 \mathrm{~T} / \mathrm{d} \alpha 2>0$, indicating that the temperature versus $\alpha$ CRTA plots of reactions fitting an Avrami-Erofeev law must yield a minimum at the reacted fraction $\alpha \mathrm{m}$. The values of $\alpha \mathrm{m}$ found for each Avrami-Erofeev model have been included in Table 2 .

On the other hand, the appearance of an inflection point at $\alpha \mathrm{m}$ would require that $\mathrm{d} 2 \mathrm{~T} / \mathrm{d} \alpha 2=$ 0 . Hence, according to Eq. (5), the following expression must hold true:

$\left[f\left(\alpha_{i}\right)\right]\left[\frac{R T}{2 E_{a}}+1\right]-f\left(\alpha_{i}\right) f\left(\alpha_{i}\right)=0$

Out of the kinetic models listed in Table 1, only diffusion ones present a solution to Eq. (8). Consequently, the temperature versus $\alpha$ CRTA plots of diffusion driven reactions are expected to show an inflection point at $\alpha$ i values.

Finally, "n-order" mechanisms yield neither minima nor maxima nor inflection points.

\subsection{Random scission models}

A random scission mechanism implies a random cleavage of the bonds along the polymer chains, producing fragments of progressively shorter length that will eventually evaporate when the size is small enough. A mathematical model for depolymerisation processes was developed by Simha-Wall [42] but it has rarely been used because the resulting $f(\alpha)$ kinetic equations cannot be expressed directly as a function of the reacted fraction, thereby making them very difficult to apply to the kinetic analysis of experimental TGA data. A recent work addressed the problem by reformulating the Simha-Wall model and proposing the following $f(\alpha)$ function for the degradation reactions governed by random scission mechanisms [39]:

$f(\alpha)=L(L-1) x(1-x) L^{-1}$

where $x$ and $L$ are the fraction of bonds broken and the minimum length of the polymer that is not volatile, respectively. Unfortunately, Eq. (9) presents a symbolic solution only when $L=2$. The problem can be sorted out by calculating numerically the $f(\alpha)$ functions for $L \neq 2$, just by giving values to both $L$ and $x$. Alternatively, a symbolic expression can be obtained by the use of a fitting function:

$(10) f(\alpha)=c n(1-\alpha) \alpha m$ 
The equation above is a modified form of the Sestak-Berggren equation [43], and it is fully capable of fitting every kinetic model in Table 1, effectively working as an umbrella that covers the different kinetic models, by merely adjusting the parameters $n, m$ and $c$ [44]. The fitting of the numerically calculated $f(\alpha)$ functions to Eq. (10) was done by means of the maximize function of Mathcad software. Table 2 lists the values that the cited $n, m$ and $c$ parameters have to take in order for Eq. (10) to match the random scission $f(\alpha)$ functions [39].

The first derivative of Eq. (10) becomes zero at $\alpha m=m /(n+m)$. Therefore, as $d 2 T / d \alpha 2>0$, the CRTA temperature versus $\alpha$ plots for reactions driven by random scission models would show a minimum like the Avrami-Erofeev models, but at lower am values as is shown in Table 2.

In order for the curves to have an inflection point, the second derivative of Eq. (4) should be equal to zero, so that Eq (8) holds true. As there is no $\alpha$ value that fulfils that condition, no inflection point should appear in the CRTA curves.

\section{Simulation of CRTA curves for different kinetic models}

The analysis above has been validated by a set of simulated $\alpha-T$ curves constructed taking into account the different kinetic models in Table 1. Fig. 3 shows a series of curves constructed assuming CRTA conditions at a constant reaction rate of $0.01 \mathrm{~min}-1$, an activation energy of $E$ $=150 \mathrm{~kJ} \mathrm{~mol}-1$ and a pre-exponential factor of $1015 \mathrm{~min}-1$. The simulation was carried out by means of the Runge-Kutta method using the Mathcad software. The curves in Fig. 3 confirm the shape analysis performed in Section 3. The curves simulated considering a diffusion kinetic model (Fig. 3a) show clearly the appearance of an inflection point while the curves drawn according to (1- $\alpha$ )n laws (Fig. 3b) present neither temperature minimums nor inflection points. It is worth noting that (1- $\alpha)$ n laws comprise both the empirical "n-order" models that lacks any physical meaning and the ideal models that were proposed assuming phase boundary controlled reactions such as the F1, R2 and R3 models that are included in Table 1. On the other hand, the curves simulated assuming random scission and Avrami-Erofeev models (Fig. $3 \mathrm{c}$ and $\mathrm{d}$ ) feature a temperature minimum at the am predicted in Table 2 . The minimum appears because at the initial stages of the reaction, the temperature raises until the predetermined reaction rate is attained. At that point, the reaction rate suddenly accelerates and the system has to cool down in order to maintain the conversion rate. In Avrami-Erofeev models, the segment previous to the acceleration can be attributed to the formation of nuclei. Once the nuclei start growing, the increase in the reaction surface induces a steady raise in the reaction rate, thus making the temperature decrease accordingly. After the temperature minimum, the coalescence that happens due to the nuclei growth provokes a reduction on the reactive surface, and the consequent diminution in the reaction rate has to be compensated by an increase in temperature [15] and [23].

A similar reasoning can be followed in the case of random scission mechanisms. The mass loss that occurs during a degradation reaction via random scission is ascribed to the evaporation of short fragments, which are most probably released after breakages close to the chain endings. The chances for those events are much lower during the initial reaction times when the polymer chains are still long and the fragments resulting from such a scission are not short enough to evaporate. Therefore, as the predetermined reaction rate is not yet achieved, the temperature keeps rising. At a certain point, when the polymer chains are sufficiently 
fragmented, the amount of material volatilized raises and the temperature must drop in order to maintain the reaction rate constant. A scheme illustrating this sequence of events is shown in Fig. 4. The minimum that appears for nucleation and random scission driven decomposition reactions is a very noticeable feature, what can be very useful for a quick identification of these mechanisms from a single CRTA curve. However, it should be taken into consideration that this procedure for determining the kinetic model can only be used if the investigated process is a single step one (that is, if the system can be described by a single set of kinetic parameters $A, E$ and $f(\alpha)$ ), or is composed by separable individual processes. The suitability of investigated processes could be checked by means of a model-free method, such as the one described by Friedman [45], which allows to determine whether the activation energy is actually independent of $\alpha$. When approaching complex reactions with overlapping events a more detailed and individualized analysis would be required. In any case, the improved resolution provided by CRTA conditions allows for a better separation of overlapping events as it has been demonstrated in literature [46].

\section{Validation of the shape analysis by real systems}

The relationship found between the shape of the CRTA curves and the reaction mechanisms have so far been proved theoretically. Next it will be tested by its application to the thermal degradation of four commercial polymers, i.e. polytetrafluoroethylene (PTFE), polyethylene (PE), poly(vinyl chloride) (PVC) and poly(1,4-butylene terephthalate) (PBT), and to evaluate the potential of this method in thermal stability studies of polymers. The thermal decomposition of PTFE, PE and PBT were studied in recent works [33], [39] and [47], where Friedman isoconversional method showed that the activation energy was independent of $\alpha$ and, hence, being single step processes suitable for the kind of analysis here proposed. The case of PVC was approached in another work, where it was shown that the degradation consists of two independent events, each due to the decomposition of polymer sequences of different tacticities [48] and [49].

Fig. $5 a$ shows the conversion and temperature plots versus time, as obtained for the thermal degradation of PBT under a linear heating rate of $1 \mathrm{~K} \mathrm{min-1.} \mathrm{In} \mathrm{these} \mathrm{experiments,} \mathrm{the} \mathrm{weight}$ loss is a direct measure of the reacted fraction while the derivative curve reflects the reaction rate. The conversion-time curve presents the sigmoidal shape that is typically obtained from conventional linear heating runs while the evolution of temperature with time follows a straight line according to the program set. On the other hand, Fig. $5 b$ shows the conversion, temperature and reaction rate as a function of time for the decomposition of the same PBT under constant rate (CRTA) experimental conditions at $8.3 \times 10-4$ min-1. It can be noticed how the reaction rate is maintained approximately constant at the selected value. Alternatively, the temperature follows an irregular profile that will depend upon the kinetic model followed by the process.

Fig. 6 shows the T- $\alpha$ plots obtained from a set of TG experiments corresponding to the thermal decomposition of PE, PTFE, PBT and PVC under a linear heating rate of $1 \mathrm{~K} \mathrm{~min}-1$. As it was expected all curves are sigmoidal in shape and no information can be immediately extracted from them. 
A single CRTA decomposition curve was also recorded for each polymer at a reaction rate of $8.3 \times 10-4$ min-1. Fig. 7 shows the temperature versus $\alpha$ plots obtained from the thermal degradation of each of them. The degradation curve of PE (Fig. 7a) shows an inflection point, which is characteristic of a diffusion mechanism. The curve obtained from the decomposition of PTFE (Fig. 7b) shows neither maximum, minimum nor inflection point, thus indicating an " $n$ order" law. On the other hand, the degradation of PBT clearly presents a minimum that appears at $\alpha=0.26$. This value is in-between the values obtained by the shape analysis (tabulated in Table 2) for random scission L2 and L3 models, indicating that a random scission mechanism drives the decomposition of PBT. The small deviation of the minimum values for the real CRTA curves as compared with those expected for the analysis of ideal models could be understood if we consider that the ideal kinetic models were proposed assuming some restricted conditions regarding geometrical consideration that shall rarely be fulfilled in real systems [44], [50], [51] and [52]. It is also interesting to relate the decomposition profile obtained for PBT (Fig. 7c) with the degradation scheme shown in Fig. 4. The positions that would correspond to the steps (1), (2) and (3) for the initial part of a polymer decomposition by a random scission mechanism have been marked in Fig. 7c. Step (1) represents the initial undegraded state. Step (2) the start of the chain breakage, when the amount of evolved material per time stands below the predetermined reaction rate and therefore the temperature keeps rising. At step (3) the chains have been shortened enough so that the fragments that evaporate significantly increase and the system are forced to cool down in order to maintain the reaction rate constant.

For the case of PVC dehydrochlorination, further clarification can be provided since it consists of two independent reactions [48] and [49]. It is clear from Fig. 6 and Fig. 7 that both events are better resolved in the CRTA curve (Fig. 7d) than in the TG one (Fig. 6d), illustrating the better resolution power of the CRTA experiment than the conventional one. The dominant event during most of conversion range is the first one that accounts up to approximately $\alpha=$ 0.8 and has the typical shape of a nucleation process. In addition, if it is taken into consideration that this first event accounts only up to $\alpha=0.8$, the equivalent value of $\alpha \mathrm{m}$ appears at about 0.40 that corresponds to the value expected for an A2 nucleation mechanism. For the second step, the CRTA curve has sigmoidal shape, suggesting a diffusion controlled kinetic model. These resulting models are in agreement with recent publications on the thermal dehydrochlorination of PVC [48] and [49].

\section{Conclusions}

Here, it has been proven that a strong relationship exists between the shape of the $\alpha-T$ CRTA curves and the kinetic model obeyed by the polymer thermal degradation process. Diffusion driven processes present an inflection point while random scission and Avrami-Erofeev models show a minimum in the temperature. Phase boundary controlled mechanisms have neither minimum nor inflection points. Since the minima in the T- $\alpha$ plots appear at much different $\alpha$ values for random scission and Avrami-Erofeev models, a single decomposition curve recorded under controlled rate conditions should be enough to unambiguously discern between polymer degradation reactions driven by different kinetic models. This asset is especially useful in the kinetic analysis of polymer degradation studies due to the complexity of the reactions and would help to identify the kinetic model without the need to resort to generic empirical 
models such as first or $\mathrm{n}$ order. Since the results obtained from a kinetic analysis are heavily dependent on the kinetic model assumed, the capacity of a single CRTA experiment to hint the model that governs a reaction just by a mere glance at the shape of the temperature-time curve, something that cannot be done in linear heating and isothermal runs, is extremely useful for any subsequent analysis and for preventing errors. The shape analysis here performed has been validated by its application to the thermal degradation of four commonly used commercial polymers: polytetrafluoroethylene (PTFE), poly(1,4-butylene terephthalate) (PBT), polyethylene (PE) and poly(vinyl chloride) (PVC). All four have been found to decompose through different mechanisms, namely "n-order", random scission, diffusion and AvramiErofeev mechanisms, respectively.

\section{Acknowledgments}

Financial support from projects TEP-03002 from Junta de Andalucía and MAT 2008-06619/MAT from the Spanish Ministerio de Ciencia e Innovación is acknowledged. The work was also supported by a JAE-Doc contract (CSIC) 
References

[1] J. Rouquerol

A general introduction to SCTA and to rate-controlled SCTA

Journal of Thermal Analysis and Calorimetry, 72 (3) (2003), pp. 1081-1086

[2] O.T. Sorensen

J. Rouquerol (Ed.), Sample controlled thermal analysis, Kluwer, Budapest (2003)

[3] G.M.B. Parkes, P.A. Barnes, E.L. Charsley

New concepts in sample controlled thermal analysis: resolution in the time and temperature domains

Analytical Chemistry, 71 (13) (1999), pp. 2482-2487

[4] E.A. Fesenko, P.A. Barnes, G.M.B. Parkes, D.R. Brown, M. Naderi

Application of SCTA to the study of the reactivity of acid-base catalysts

Journal of Thermal Analysis and Calorimetry, 72 (3) (2003), pp. 1103-1105

[5] O.T. Sorensen

SCTA and ceramics

Journal of Thermal Analysis and Calorimetry, 72 (3) (2003), pp. 1093-1095

[6] T. Arii, K. Terayama, N. Fujii

Controlled-rate thermal analysis - Study of the process of super hard material debinding

Journal of Thermal Analysis, 47 (6) (1996), pp. 1649-1661

[7] L.A. Perez-Maqueda, J.M. Criado, C. Real, J. Subrt, J. Bohacek

The use of constant rate thermal analysis (CRTA) for controlling the texture of hematite obtained from the thermal decomposition of goethite

Journal of Materials Chemistry, 9 (8) (1999), pp. 1839-1845

[8] M.D. Alcalá, J.M. Criado, F.J. Gotor, C. Real

beta-SiALON obtained from carbothermal reduction of kaolinite employing sample controlled reaction temperature (SCRT)

Journal of Materials Science, 41 (7) (2006), pp. 1933-1938

[9] E.A. Fesenko, P.A. Barnes, G.M.B. Parkes, E.A. Dawson, M.J. Tiernan 
Catalyst characterisation and preparation using sample controlled thermal techniques - high resolution studies and the determination of the energetics of surface and bulk processes

Topics in Catalysis, 19 (3-4) (2002), pp. 283-301

[10] E.A. Dawson, G.M.B. Parkes, P.A. Barnes, M.J. Chinn, L.A. Pears, C.J. Hindmarsh

A study of evolved gas control and its effect on carbon yield during the activation of carbon fibres by controlled rate methods

Carbon, 40 (15) (2002), pp. 2897-2903

[11] O. Monnereau, L. Tortet, P. Llewellyn, F. Rouquerol, G. Vacquier

Synthesis of $\mathrm{Bi} 2 \mathrm{O} 3$ by controlled transformation rate thermal analysis: a new route for this oxide?

Solid State lonics, 157 (1-4) (2003), pp. 163-169

[12] P. Llewellyn, J. Rouquerol

SCTA and adsorbents

Journal of Thermal Analysis and Calorimetry, 72 (3) (2003), pp. 1099-1101

[13] F. Chehimi-Moumen, P. Llewellyn, F. Rouquerol, G. Vacquier, D.B. Hassen-Chehimi, M. Ferid, M. Trabelsi-Ayadi

Constant transformation rate thermal analysis of $\mathrm{HGdP2O}$ center dot $3 \mathrm{H}(2) \mathrm{O}$

Journal of Thermal Analysis and Calorimetry, 82 (3) (2005), pp. 783-789

[14] G. Agarwal, R.F. Speyer

R.F. Lobo, J.S. Beck, S.L. Suib, D.R. Corbin, M.E. Davis, L.E. Iton, S.I. Zones (Eds.), Microporous and macroporous materials: gas sensing behavior of porous $\mathrm{ZnO}$ varistors densified via rate controlled sintering (1996), pp. 427-432

[15] J.M. Criado, L.A. Perez-Maqueda

Sample controlled thermal analysis and kinetics

Journal of Thermal Analysis and Calorimetry, 80 (1) (2005), pp. 27-33

[16] L.A. Perez-Maqueda, J.M. Criado, F.J. Gotor

Controlled rate thermal analysis commanded by mass spectrometry for studying the kinetics of thermal decomposition of very stable solids

International Journal of Chemical Kinetics, 34 (3) (2002), pp. 184-192

[17] S. Yamada, N. Koga 
Kinetics of the thermal decomposition of sodium hydrogen carbonate evaluated by controlled rate evolved gas analysis coupled with thermogravimetry

Thermochimica Acta, 431 (1-2) (2005), pp. 38-43

[18] T. Arii, S. Ichihara, H. Nakagawa, N. Fujii

A kinetic study of the thermal decomposition of polyesters by controlled-rate thermogravimetry

Thermochimica Acta, 319 (1-2) (1998), pp. 139-149

[19] T. Arii, Y. Masuda

The effect of humidity on thermal decomposition of terephthalate polyester

Journal of Analytical and Applied Pyrolysis, 71 (2) (2004), pp. 525-536

[20] M. Reading

The Kinetics of heterogeneous solid-state decomposition reactions. A new way forward Thermochimica Acta, 135 (1988), pp. 37-57

[21] M. Reading

P.K. Gallagher (Ed.), Handbook of thermal analysis and calorimetry: controlled rate thermal analysis and related techniques, Elsevier, Amsterdam (1998), pp. 423-443

[22] J.M. Criado, L.A. Perez-Maqueda, M.J. Dianez, P.E. Sanchez-Jimenez

Development of a universal constant rate thermal analysis system for being used with any thermoanalytical instrument

Journal of Thermal Analysis and Calorimetry, 87 (1) (2007), pp. 297-300

[23] M.J. Tiernan, P.A. Barnes, G.M.B. Parkes

New approach to the investigation of mechanisms and apparent activation energies for the reduction of metal oxides using constant reaction rate temperature-programmed reduction

Journal of Physical Chemistry B, 103 (2) (1999), pp. 338-345

[24] E.A. Dawson, G.M.B. Parkes, P.A. Barnes, M.J. Chinn, P.R. Norman

A study of the activation of carbon using sample controlled thermal analysis

Journal of Thermal Analysis and Calorimetry, 56 (1) (1999), pp. 267-273

[25] N. Koga, J.M. Criado

The influence of mass transfer phenomena on the kinetic analysis for the thermal decomposition of calcium carbonate by constant rate thermal analysis (CRTA) under vacuum 
International Journal of Chemical Kinetics, 30 (10) (1998), pp. 737-744

[26] J.M. Criado, F.J. Gotor, A. Ortega, C. Real

The New method of constant rate thermal-analysis (Crta) - application to discrimination of the kinetic-model of solid-state reactions and the synthesis of materials

Thermochimica Acta, 199 (1992), pp. 235-238

[27] J.M. Criado, J. Morales

Thermal decomposition reactions of solids controlled by diffusion and phase-boundary processes - possible misinterpretation from thermogravimetric data

Thermochimica Acta, 19 (3) (1977), pp. 305-317

[28] J.M. Criado, J. Morales

Defects of thermogravimetric analysis for discerning between 1st order reactions and those taking place through Avrami-Erofeevs mechanism

Thermochimica Acta, 16 (3) (1976), pp. 382-387

[29] L.A. Perez-Maqueda, J.M. Criado, F.J. Gotor, J. Malek

Advantages of combined kinetic analysis of experimental data obtained under any heating profile

Journal of Physical Chemistry A, 106 (12) (2002), pp. 2862-2868

[30] J.M. Criado, A. Ortega

Remarks on the discrimination of the kinetics of solid-state reactions from a single nonisothermal trace

Journal of Thermal Analysis, 29 (6) (1984), pp. 1225-1236

[31] A. Khawam, D.R. Flanagan

Solid-state kinetic models: basics and mathematical fundamentals

Journal of Physical Chemistry B, 110 (35) (2006), pp. 17315-17328

[32] N. Koga, H. Tanaka

A physico-geometric approach to the kinetics of solid-state reactions as exemplified by the thermal dehydration and decomposition of inorganic solids

Thermochimica Acta, 388 (1-2) (2002), pp. 41-61 
[33] P.E. Sanchez-Jimenez, L.A. Perez-Maqueda, A. Perejon, J.M. Criado

Combined kinetic analysis of thermal degradation of polymeric materials under any thermal pathway

Polymer Degradation and Stability, 94 (11) (2009), pp. 2079-2085

[34] L. Nunez, M. Villanueva, B. Rial, M.R. Nunez, L. Fraga

Thermal stability of epoxy systems BADGE ( $n=0) / 1,2-D C H$ and BADGE $(n=0) / 1,2-$ DCH/vinylcyclohexene dioxide

Journal of Thermal Analysis and Calorimetry, 70 (1) (2002), pp. 75-84

[35] J.M. Salla, J.M. Morancho, A. Cadenato, X. Ramis

Non-isothermal degradation of a thermoset powder coating in inert and oxidant atmospheres

Journal of Thermal Analysis and Calorimetry, 72 (2) (2003), pp. 719-728

[36] C.X. Guan, L.Q. Li, D.H. Chen, Z.Y. Gao, W.B. Sun

Thermal behavior and thermal decomposition study of porphyrin polymers containing different spacer groups

Thermochimica Acta, 413 (1-2) (2004), pp. 31-38

[37] P. Budrugeac, E. Segal, L.A. Perez-Maqueda, J.M. Criado

The use of the IKP method for evaluating the kinetic parameters and the conversion function of the thermal dehydrochlorination of PVC from non-isothermal data

Polymer Degradation and Stability, 84 (2) (2004), pp. 311-320

[38] B. Jankovic, L. Kolar-Anic, I. Smiciklas, S. Dimovic, D. Arandelovic

The non-isothermal thermogravimetric tests of animal bones combustion. Part. I. Kinetic analysis

Thermochimica Acta, 495 (1-2) (2009), pp. 129-138

[39] P.E. Sanchez-Jimenez, L.A. Perez-Maqueda, A. Perejon, J.M. Criado

A new model for the kinetic analysis of thermal degradation of polymers driven by random scission

Polymer Degradation and Stability, 95 (5) (2010), pp. 733-739

[40] L.A. Perez-Maqueda, J.M. Criado, J. Subrt, C. Real

Synthesis of acicular hematite catalysts with tailored porosity

Catalysis Letters, 60 (3) (1999), pp. 151-156 
[41] M.J. Dianez, L.A. Perez-Maqueda, J.M. Criado

Direct use of the mass output of a thermobalance for controlling the reaction rate of solidstate reactions

Review of Scientific Instruments, 75 (8) (2004), pp. 2620-2624

[42] R. Simha, L.A. Wall

Kinetics of Chain Depolymerization

Journal of Physical Chemistry, 56 (6) (1952), pp. 707-715

[43] J. Sestak, G. Berggren

study of the kinetics of the mechanism of solid-state reactions at increased temperature

Thermochimica Acta, 3 (1971), pp. 1-12

[44] L.A. Perez-Maqueda, J.M. Criado, P.E. Sanchez-Jimenez

Combined kinetic analysis of solid-state reactions: a powerful tool for the simultaneous determination of kinetic parameters and the kinetic model without previous assumptions on the reaction mechanism

Journal of Physical Chemistry A, 110 (45) (2006), pp. 12456-12462

[45] H.L. Friedman

Kinetics of thermal degradation of char-forming plastics from thermogravimetry

Application to Phenolic Plastic Journal of Polymer Science Part C-Polymer Symposium (6PC) (1964), pp. 183-195

[46] P.E. Sanchez-Jimenez, L.A. Perez-Maqueda, J.E. Crespo-Amoros, J. Lopez, A. Perejon, J.M. Criado

Quantitative characterization of multicomponent polymers by sample-controlled thermal analysis

Analytical Chemistry, 82 (21) (2010), pp. 8875-8880

[47] P.E. Sanchez-Jimenez, L.A. Perez-Maqueda, A. Perejon, J.M. Criado

Generalized kinetic master plots for the thermal degradation of polymers following a random scission mechanism

Journal of Physical Chemistry A, 114 (30) (2010), pp. 7868-7876

[48] P.E. Sanchez-Jimenez, A. Perejon, J.M. Criado, M.J. Dianez, L.A. Perez-Maqueda

Kinetic model for thermal dehydrochlorination of poly(vinyl chloride) 
Polymer, 51 (17) (2010), pp. 3998-4007

[49] A. Perejon, P.E. Sanchez-Jimenez, J.M. Criado, L.A. PerezMaqueda

Kinetic analysis of complex solid state reactions. A new deconvolution procedure

Journal of Physical Chemistry B (2011) http://dx.doi.org/10.1021/jp110895z

[50]N. Koga, J.M. Criado

Influence of the particle size distribution on the CRTA curves for the solid-state reactions of interface shrinkage

Journal of Thermal Analysis, 49 (3) (1997), pp. 1477-1484

[51] N. Koga, J.M. Criado

Kinetic analyses of solid-state reactions with a particle-size distribution

Journal of the American Ceramic Society, 81 (11) (1998), pp. 2901-2909

[52] E.A. Cooper, T.O. Mason

Mechanism of La2cuo4 solid-state powder reaction by quantitative Xrd and impedance spectroscopy

Journal of the American Ceramic Society, 78 (4) (1995), pp. 857-864 


\section{Figure captions}

Figure 1. Trend of temperature, mass lost and reaction rate during a thermal decomposition reaction under different heating methods. This figure has been adapted from the one first published by Reading [20] and [21].

Figure 2. Scheme showing the differences between a conventional heating control system (a) and the more sophisticated controlled rate thermal analysis (b).

Figure 3. $T-\alpha$ plots corresponding to simulated CRTA curves (reaction rate $0.01 \mathrm{~min}-1$ ) constructed by using the following kinetic parameters: activation energy of $150 \mathrm{~kJ} \mathrm{~mol}-1$, a pre-exponential factor of 1015 min-1 and (a) diffusion, (b) phase boundary contraction, (c) random scission; and (d) Avrami-Erofeev kinetic models.

Figure 4. Scheme illustrating the initial steps of polymer decomposition by a random scission mechanism

Figure 5. Experimental conversion (solid line), temperature (squares) and reaction rate (dotted line) against time curves obtained for the thermal decomposition of PBT under (a) a linear heating rate of $1 \mathrm{~K} \mathrm{~min}-1$ and (b) constant rate conditions at $8.3 \times 10-4 \mathrm{~min}-1$.

Figure 6. Experimental temperature versus $\alpha$ plots corresponding to the thermal decomposition obtained under constant linear heating at $1 \mathrm{~K} \mathrm{min-1}$ of (a) polyethylene, (b) polytetrafluoroethylene, (c) poly(1,4-butylen)terephthalate; and (d) polyvinyl chloride.

Figure 7. Experimental temperature versus $\alpha$ plots corresponding to the thermal decomposition under CRTA conditions of (a) polyethylene, (b) polytetrafluoroethylene, (c) poly(1,4-butylen)terephthalate; and (d) polyvinyl chloride. The kinetic model for each reaction as deduced from the shape of the curves is also marked under each plot. 
Table 1

Table 1. $f(\alpha)$ kinetic functions for the most widely used kinetic models, including the newly proposed random scission model

\begin{tabular}{|c|c|c|c|c|}
\hline Mechanism & Symbo & $\mathrm{ff}(\alpha)$ & $\mathbf{f}^{\prime}(\alpha)$ & $f^{\prime \prime}(\alpha)$ \\
\hline $\begin{array}{l}\text { Phase boundary controlled } \\
\text { reaction (contracting area) }\end{array}$ & R2 & $(1-\alpha)^{1 / 2}$ & $\frac{-1}{2(1-\alpha)^{1 / 2}}$ & $\frac{-1}{4(1-\alpha)^{3 / 2}}$ \\
\hline \begin{tabular}{|l|} 
Phase boundary controlled \\
reaction \\
volume)
\end{tabular} & & $(1-\alpha)^{2 / 3}$ & $\frac{-2}{3(1-\alpha)^{1 / 3}}$ & $\frac{-2}{9(1-\alpha)^{4 / 3}}$ \\
\hline 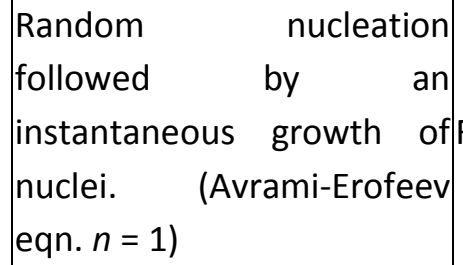 & $\mathrm{F} 1$ & $(1-\alpha)$ & -1 & 0 \\
\hline $\begin{array}{l}\text { Random nucleation and } \\
\text { growth of nuclei through } \\
\text { different nucleation and } \\
\text { nucleus growth models. } \\
\text { (Avrami-Erofeev eqn } \neq 1 \text {.) }\end{array}$ & & $\begin{array}{l}n(1-\alpha)[- \\
\ln (1-\alpha)]^{1^{-}} \\
1 / n\end{array}$ & $\frac{n \ln (1-\alpha)+n-1}{\llbracket-\ln (1-\alpha)]^{1 / \alpha}}$ & $\frac{-n+1+\frac{1-1 / n}{\operatorname{Inn}(1-a)}}{(1-\alpha)[-\ln (1-\alpha)]^{1 / 3 n}}$ \\
\hline Two-dimensional diffusion & D2 & $-1 / \ln (1-\alpha)$ & $\frac{-1}{(1-\alpha)[\ln (1-\alpha)]^{2}}$ & $\frac{-2-\ln (1-\alpha)}{(1-\alpha)^{2}[\ln (1-\alpha)]^{3}}$ \\
\hline $\begin{array}{l}\text { Three-dimensional } \\
\text { diffusion (Jander equation) }\end{array}$ & D3 & $\frac{3(1-\alpha)^{2 / 3}}{2\left[1-(1-\alpha)^{1 / 3}\right]}$ & $\frac{1 / 2-(1-\alpha)^{-1 / 3}}{\left[1-(1-\alpha)^{1 / 3}\right]^{2}}$ & $\left(\frac{3(1-\alpha)^{-1 / 3}-(1-\alpha)^{-2 / 3}-1}{3(1-\alpha)^{2 / 3}\left[1-(1-\alpha)^{1 / 3}\right]^{3}}\right)$ \\
\hline \begin{tabular}{|l|} 
Three-dimensional \\
diffusion (Ginstling- \\
Brounshtein equation)
\end{tabular} & D4 & $\begin{array}{l}(1-2 \alpha / 3)-(1 \\
-\alpha)^{2 / 3}\end{array}$ & $\left|\frac{-(1-\alpha)^{-4 / 3}}{2\left[(1-\alpha)^{-1 \beta^{3}}-1^{2}\right]}\right|$ & $\frac{2\left[(1-\alpha)^{1 / 3}-1\right]+1}{3(1-\alpha)^{8 / 3}\left[(1-\alpha)^{-1 / 3}-1\right]^{3}}$ \\
\hline Random Scission $L=2$ & L2 & $2(\alpha 1 / 2-\alpha)$ & $\frac{1}{a^{1 / 2}}-2$ & $\frac{1}{2 \alpha^{3 / 2}}$ \\
\hline Random Scission $L>2$ & $L>2$ & $\begin{array}{l}\text { No symbolic } \\
\text { solution }\end{array}$ & $\begin{array}{l}\text { No symbolic } \\
\text { solution }\end{array}$ & No symbolic solution \\
\hline
\end{tabular}




\section{Table 2}

Table 2. Position of the minimum on the temperature- $\alpha$ plots for the case of Avrami-Erofeev and random scission models together with the values the parameters $\mathrm{c}, \mathrm{n}$ and $\mathrm{m}$ have to take in order to make Eq. (10) match the corresponding ideal models.

\begin{tabular}{|l|l|l|l|l|}
\hline Symbol & $a_{\mathrm{m}}$ & $\mathrm{n}$ & $\mathrm{m}$ & $\mathrm{c}$ \\
\hline A2 & 0.393 & 0.807 & 0.515 & 2.081 \\
\hline A3 & 0.486 & 0.751 & 0.695 & 3.204 \\
\hline A4 & 0.528 & 0.725 & 0.787 & 4.340 \\
\hline L2 & 0.250 & 1.119 & 0.400 & 1.204 \\
\hline L3 & 0.273 & 1.057 & 0.396 & 2.080 \\
\hline L4-L8 & 0.275 & $1.039 \rightarrow 1.017$ & $0.394 \rightarrow 0.386$ & $2.93 \rightarrow 6.24$ \\
\hline
\end{tabular}


Figure 1

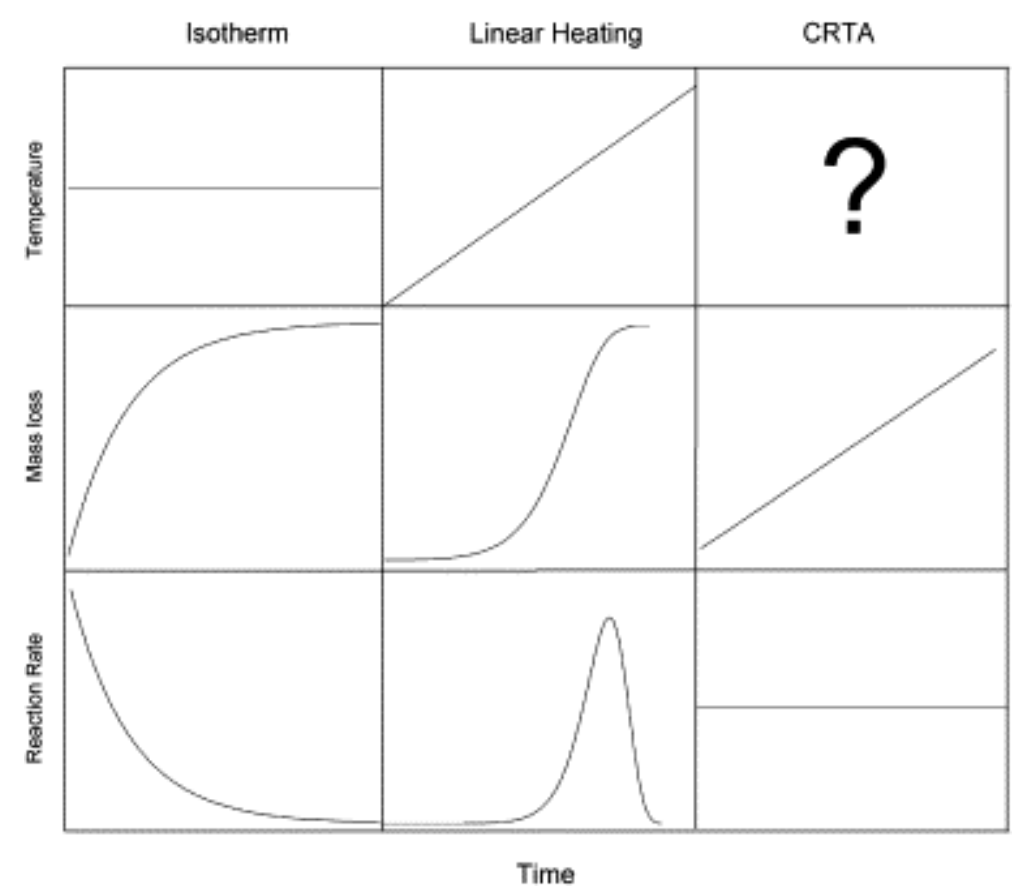


Figure 2
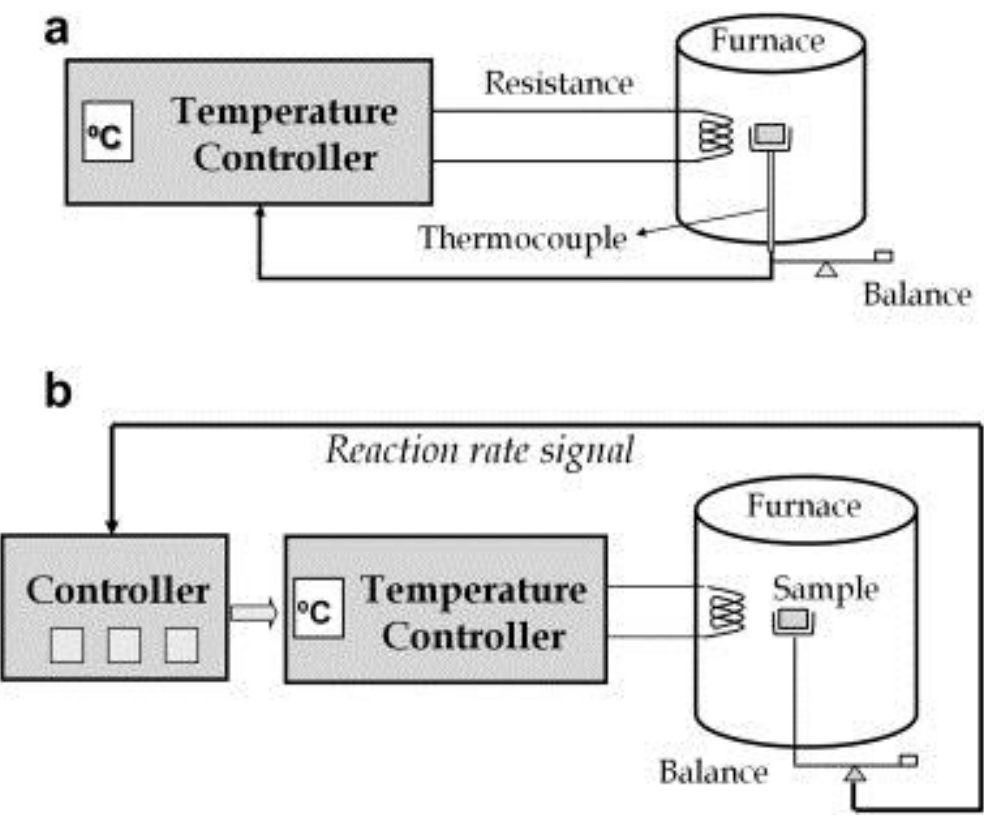
Figure 3
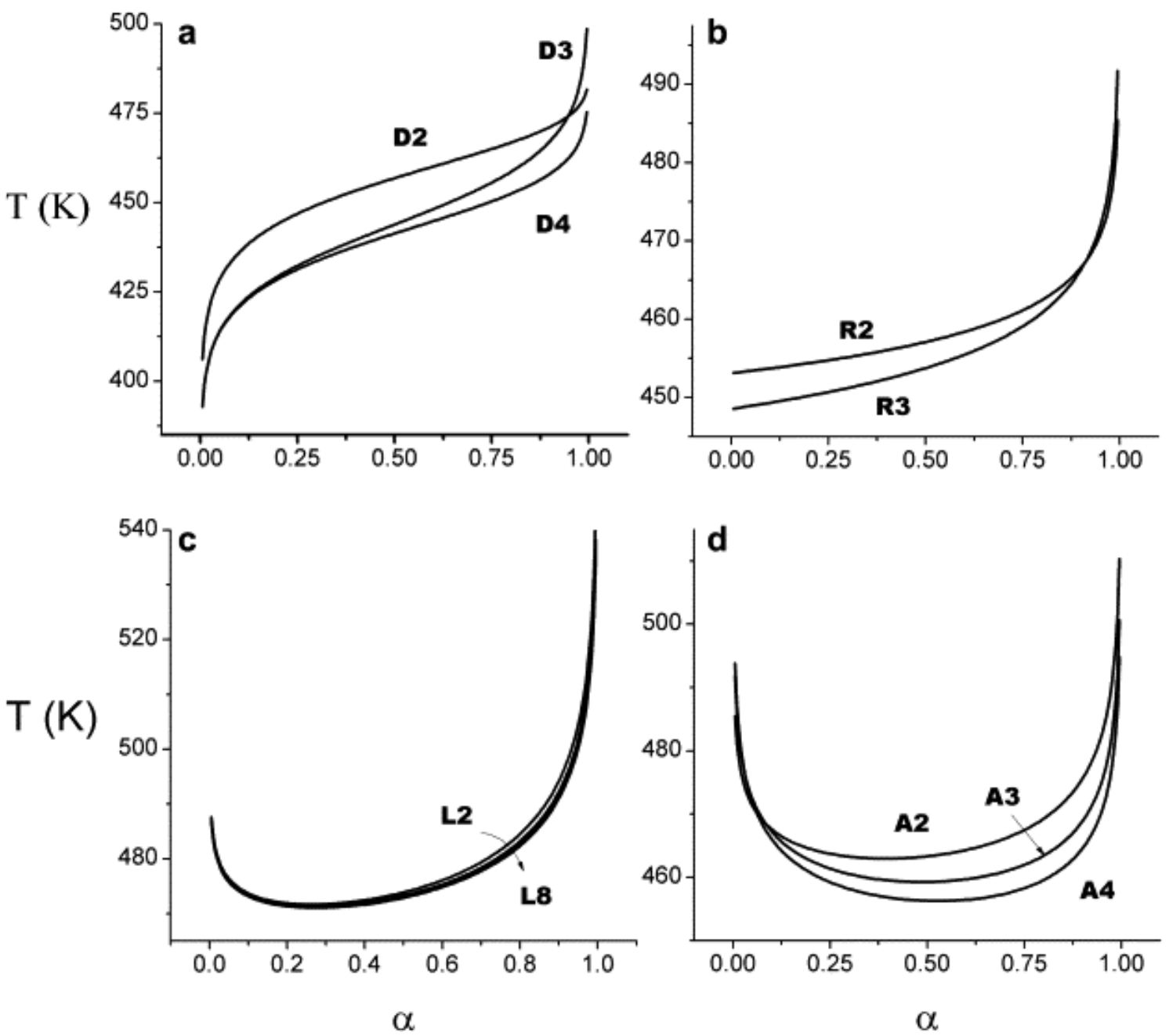
Figure 4

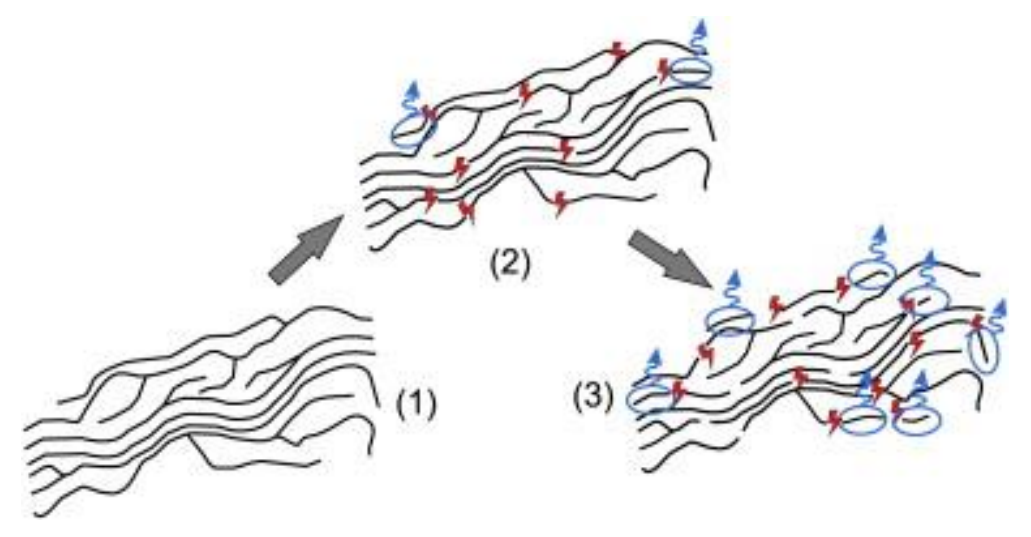


Figure 5
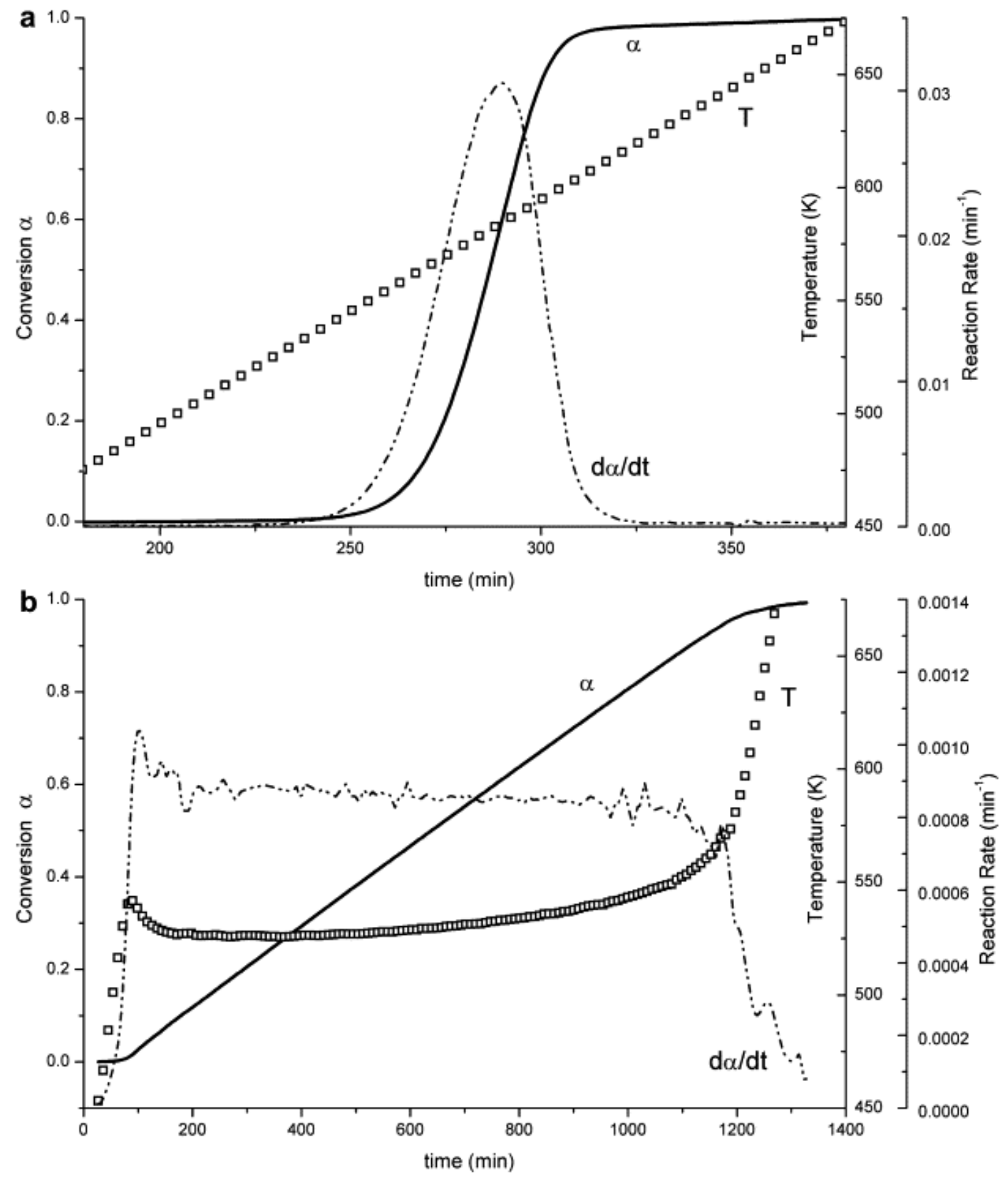
Figure 6
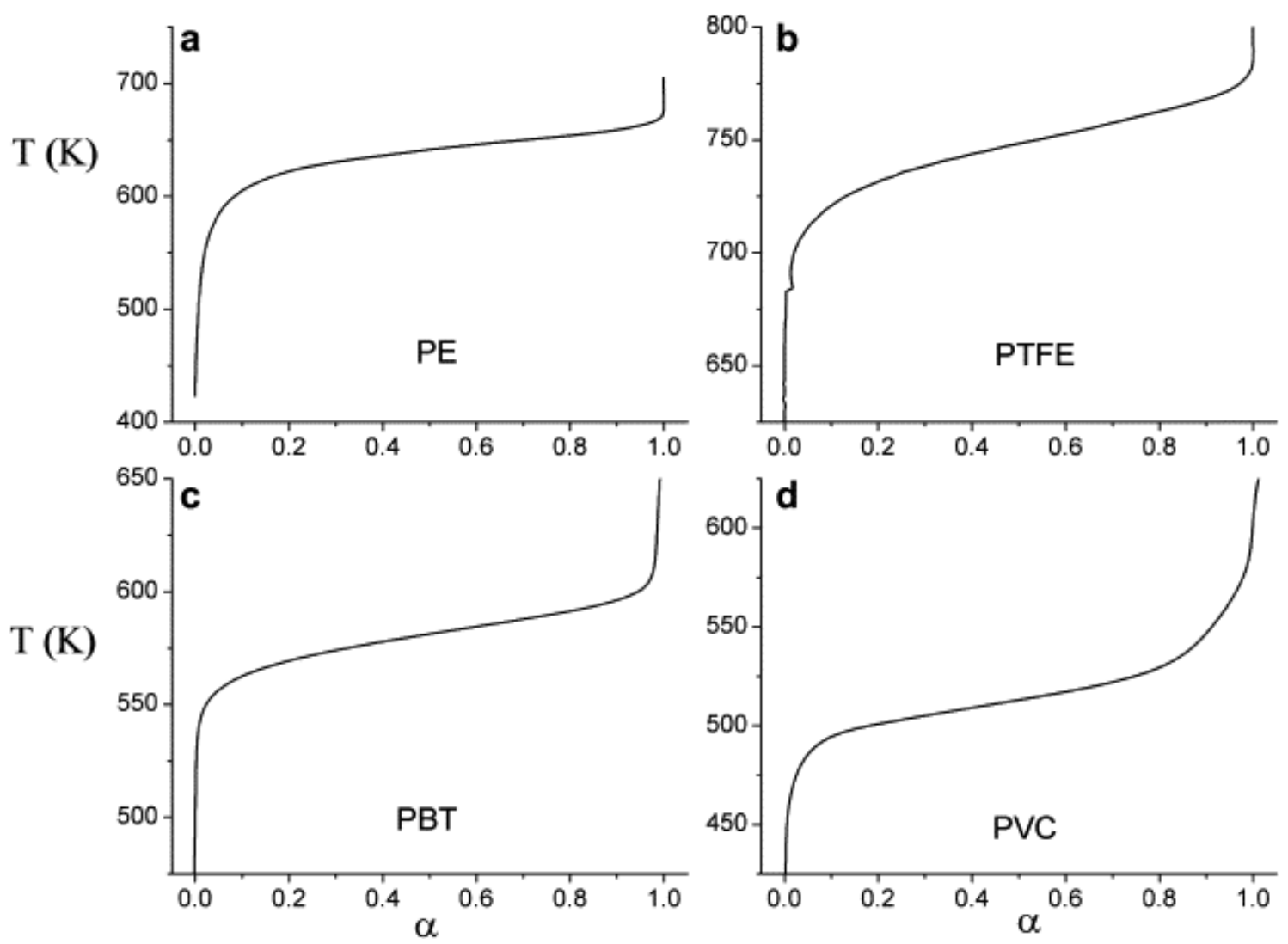
Figure 7
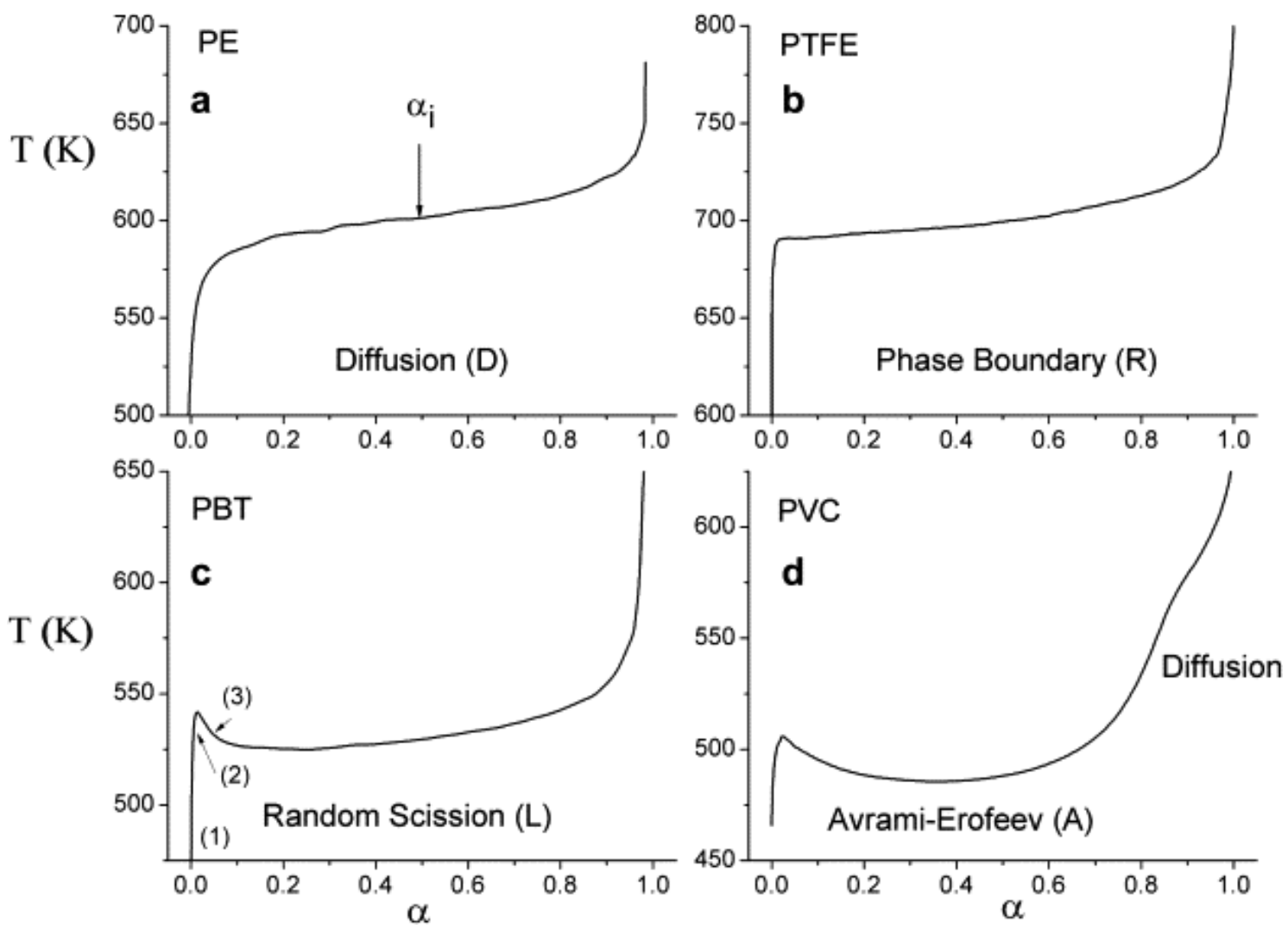